\title{
ESTRATÉGIAS DE GRADAÇÃO EM COMENTÁRIOS DE UM VÍDEO NO YOUTUBE
}

\section{GRADUATION STRATEGIES IN YOUTUBE VIDEO COMMENTS}

\author{
Rodrigo Esteves de Lima-Lopes (Unicamp/CNPQ) \\ rll307@unicamp.br \\ Carolina Palma de Sousa Arruda (Unicamp) \\ carolpalma203@gmail.com
}

\begin{abstract}
RESUMO: Tem-se por objetivo estudar a gradação em um corpus composto por 5.133 comentários extraídos de um vídeo no YouTube apoiando um candidato à presidência da república de extrema-direita. O referencial teórico está na Linguística Sistêmico-Funcional (LSF), uma abordagem socialmente orientada à análise da linguagem, e no sistema de avaliatividade, uma teoria advinda da LSF e que busca compreender os processos avaliativos da linguagem. Como método para coleta e análise de dados, utilizou-se a Linguística do Corpus, com especial ênfase para scripts na linguagem de programação $R$ para a raspagem $e$ análise de dados. As estratégias foram quantificadas e submetidas a testes de correlação (Pearson-R). Os resultados mostram um forte protagonismo do termo mito, apresentando em diversas estratégias de gradação cujos padrões linguísticos foram mapeados, indicando-se como as estratégias coocorrem. Essa pesquisa traz contribuições relevantes para o estudo da avaliatividade e da gradação no discurso político cotidiano da internet.
\end{abstract}

PALAVRAS-CHAVE: Linguística Sistêmico-Funcional; sistema de avaliatividade; Linguística de Corpus; comunicação mediada por computador; programação em R.

ABSTRACT: We aim at studying the graduation in a corpus of 5,133 comments extracted from a video on YouTube supporting a far-right candidate for the last Brazilian presidential election. Systemic-Functional Linguistics (SFL), a socially oriented approach to language analysis, and the appraisal system, a theory that comes from SFL and seeks to understand the evaluative processes of language, are our theoretical framework. Corpus Linguistics was the main methodological framework for collecting and analysing data. Scripts in the $R$ programming language were written for data scraping and analysis. The strategies were quantified and correlation tests (Pearson-R) were applied. Results show a strong protagonism of the term mito, present in several graduating strategies whose linguistic patterns were mapped. Correlation tests indicated how the strategies co-occur in the comments. This research brings relevant contributions to the study of graduation in the daily political discourse of the internet. 
KEYWORDS: Systemic-Functional Linguistics; appraisal system; Corpus Linguistics; computer-mediated communication; R programming language.

\section{Introdução}

Este artigo tem por objetivo estudar os recursos de gradação em um corpus de comentários de internautas extraídos de um vídeo no YouTube. Dentro do contexto da Linguística Sistêmico-Funcional (HALLIDAY, 1978, 2013), doravante LSF, o sistema de gradação poderia ser definido como um subsistema da avaliatividade (MARTIN; WHITE, 2005), cuja função estaria relacionada à amplificação ou mitigação de processos de julgamento e heteroglossia dentro do subsistema de força (ver seção de fundamentação teórica a seguir para maiores detalhes). A análise proposta neste artigo busca especificamente refletir sobre como a palavra mito, de forma a compreender como os recursos de gradação são utilizados de modo a construir uma imagem na polaridade positiva, está largamente utilizada como forma de avaliação favorável a um dos candidatos à presidência durante a eleição em 2018 (MAZUI; CALGARO, 2020).

Em outras palavras, buscamos entender se o uso de MITO - grafado em letras maiúsculas - é mais ou menos frequente que o uso de mito - grafado exclusivamente em letras minúsculas —, ou mesmo que BolsoMito - palavra na qual mito é escrito junto ao sobrenome do candidato - , e se essas e outras estratégias coocorrem de forma significativa nos comentários.

Este trabalho se apoia na Linguística Sistêmico-Funcional (doravante LSF) (HALLIDAY, 1978, 2013), mais especificamente no sistema de avaliatividade (ALBA-JUEZ; THOMPSON, 2014; MARTIN; WHITE, 2005) como base teórica e na Linguística do Corpus (doravante LC) (BAKER, 2009; BERBER-SARDINHA, 2004; BONELLI, 2012) como abordagem metodológica para coleta e análise de dados.

Há diversos estudos de avaliatividade em língua portuguesa. Eles estão relacionados a reflexões teóricas sobre a natureza desse sistema em nosso idioma (ALMEIDA, 2011a; SOUZA, 2011; VIAN JR, 2009; VIAN JR; LIMA-LOPES, 2005), a julgamento em artigos jornalísticos de editoria política (CABRAL, 2007, 2011), a discursos de professores (ALMEIDA, 2011b), a obras literárias (VIAN JR., 2011), a atitude e julgamento em mídias sociais (LIMA-LOPES, 2018; LIMA-LOPES; PIMENTA, 2017) e a blogs (ALMEIDA, 2018). Entretanto, todas são investigações que se preocupam com marcas lexicais e 
semântico-discursivas de avaliatividade, não havendo trabalhos sobre o que Crystal (1997) chama de variação discursiva tipográfica na internet, graficamente sinalizando avaliações em contextos virtuais. Tal fenômeno, segundo o autor, abarcaria questões como a repetição de letras ou sílabas, sua representação em letras maiúsculas ou minúsculas, além de abreviações típicas da escrita em ambiente digital.

Acreditamos que tais marcas sejam valiosas para o estudo da avaliatividade, uma vez que, como bem coloca Crystal (1997), eles podem estar relacionados a questões tanto de etiqueta comunicacional (LÉVY, 1995), como também dos recursos de expressão possibilitados pela comunicação mediada por computador (O'REGAN; MOMPEAN; DESMET, 2010), incluindo significados como ênfase, polaridade, expressividade entre outros. Consideramos que grafias como Mito, MITO ou Mitooooo, todas retiradas do corpus desta pesquisa, podem causar diferentes impactos quando presentes de forma isolada ou associadas nos diversos comentários. Apesar de Crystal (1997) não discutir em qual nível de análise esse tipo de transformação linguística se dá, acreditamos que o estudo de como tais estratégias se aplicam seja uma importante contribuição para compreender como tais processos podem intensificar a já inerente avaliação presente na palavra mito. Ademais, apesar de tais marcas apontarem importantes elementos no estabelecimento dos processos de gradação (MARTIN; WHITE, 2005, p. 37), estudos sobre o tema inexistem em nosso idioma.

Em vista disso, este trabalho busca fomentar a discussão a partir de duas perspectivas. A primeira estaria relacionada ao espaço no qual as interações estão relacionadas, o midiativismo digital (BRAIGHI; CÂMARA, 2018) e sua análise por meio da LSF e do sistema de avaliatividade. Apesar de haver alguns estudos que discutem a questão (GABARDO; LIMA-LOPES, 2018; LIMA-LOPES, 2018; LIMA-LOPES; GABARDO, 2019; LIMA-LOPES; PIMENTA, 2017), seu foco principal acaba sendo o campo semântico de julgamento presente nas diferentes mídias sociais. A outra está na utilização de abordagens baseadas no processamento de corpora para a análise da avaliatividade em língua portuguesa, algo relevante se pensarmos que a maioria dos estudos realizados em nosso idioma são de caráter qualitativo (ALMEIDA; VIAN JR, 2018).

Se, por um lado, a popularização da internet, bem como a autonomia que ela fornece aos seus usuários, trouxe mudanças no modo como movimentos sociais são organizados e questões são colocadas, propiciando a vozes antes silenciadas a oportunidade de serem ouvidas (CASTELLS, 2015; LÉVY, 2012), por outro, tais mídias permitem o surgimento de discursos conservadores e preconceituosos em massa (HOFFMAN, 1996). É o que LimaLopes (2018) observa quando analisa os comentários enviados a uma postagem no Facebook 
referente à ExpoQueer, uma exibição de arte patrocinada por uma instituição financeira, responsável, também, pela divulgação nas mídias sociais. Seus resultados mostram que as postagens tendem a se repetir, sendo que as críticas não se baseiam em uma análise das obras expostas, mas em um copiar e colar de comentários anteriores que pré-julgam as entidades envolvidas. Seus resultados corroboram o trabalho de Sovignon \& Roithmeier (2013), para quem a repetição parcial de mensagens ou de estratégias avaliativas é um recurso importante na autoidentificação no contexto da Comunicação Mediada por Computador. De fato, em regimes de pós-verdade, como aponta Bentes (2018), há um sistema de crenças que opera nas comunidades da internet, nas quais fatos são deixados de lado e há o reforço de preconceitos. Isso talvez possa explicar a razão pela qual as pessoas começam a se identificar como produtoras de conteúdo, validando preconceitos e reforçando discursos conservadores.

Entre as mídias em que há produções desse tipo de discurso e engajamento de usuários, escolhemos estudar o YouTube. Tal escolha se dá principalmente pelo fato de ele estar entre as mídias que têm recebido menor atenção por pesquisadores brasileiros em nossa área (LIMA-LOPES; CÂMARA, 2019). Silva (2018) aponta que a plataforma é um agente na propagação de mensagens em massa. $\mathrm{O}$ autor percebe que, além dos canais oficiais dos postulantes à presidência, encontram-se vários usuários que, apesar de não pertencerem formalmente à classe política, disseminam em seus vídeos seus pontos de vista, bem como o apoio a certos candidatos. Silva (2018) conclui que a autointitulada direita se configura pela centralidade em personalidades conservadoras cristãs, identificando-se por meio de detalhes indiretos e nas relações que constituem entre si.

Por fim, este artigo está organizado da seguinte forma. Na seção seguinte, trazemos à baila discussões sobre a LSF, seguida dos procedimentos metodológicos que nortearam esta pesquisa, incluindo a coleta e os procedimentos para a análise dos dados. A Seção 4 discute os resultados desta pesquisa, aos quais seguem as considerações finais.

\section{A Linguística Sistêmico-Funcional e o sistema de avaliatividade}

Nossas bases teóricas estão na LSF (HALLIDAY, 1978, 2013), mais especificamente no sistema de avaliatividade (MARTIN; WHITE, 2005; THOMPSON; ALBA-JUEZ, 2014). A LSF é uma teoria de caráter social que vê a linguagem como um conjunto de escolhas largamente influenciado pelas necessidades de expressão em um dado contexto. Tal abordagem une forma e significado de maneira a discutir como escolhas socialmente significativas são realizadas e instanciadas nos processos de significação (HALLIDAY; 
MATTHIESSEN, 2014; KRESS, 2010).

Na LSF, os textos são instanciados a partir das variáveis do seu contexto de produção, seja ele imediato ou social. Halliday (1978) desenvolve um modelo baseado em uma tríade de funções que não se caracteriza como um conjunto de regras a serem refinadas. Elas são variáveis que orientam a constituição do ato comunicativo desde as esferas mais abstratas, como é o contexto de cultura, ou o mais relacionado ao momento imediato de realização do texto, ou contexto de situação (EGGINS, 2004).

Para Halliday (1978), o contexto de situação (ou registro) não é uma simples influência do contexto nas escolhas que realizamos ao nos comunicarmos. Ele deve ser definido como uma característica semântica relacionada a uma dimensão cultural da instanciação da linguagem, que varia funcionalmente a partir dele (MATTHIESSEN, 1993). Logo, nesse modelo, a construção de significados está intrinsecamente relacionada às experiências que propiciam o conhecimento discursivo necessário para interpretar e produzir sentido.

Logo, a linguagem inevitavelmente ocorre em contexto. Para Matthiessen (1993), a língua é um sistema de sistemas, que se organiza semanticamente de forma estratificada e multissistêmica. Esses sistemas possuem formas específicas de realização, o que caracteriza a língua como um construto multifuncional e multissignificativo (HALLIDAY; HASAN, 1991). Uma diferença entre esse modelo e outros modelos funcionais é que ele não tem como elemento-chave a ideia de que há uma função que prevalece no ato comunicativo. Os diversos significados são instanciados de forma concomitante e com igual importância. Isso enriquece nossa perspectiva de compreensão e análise (BEAUGRANDE, 1993), fazendo com que o modelo da LSF veja a língua como um sistema trinocular de construção de significados (IEDEMA, 2003), levando em conta as três variáveis de registro (campo, modo e relações) de forma simultânea e com igual importância. Assim, de acordo com Eggins e Martin (1997), as variáveis de registro, definidas por Halliday (HALLIDAY; HASAN, 1991) e posteriormente reescritas pelos autores, poderiam ser definidas da seguinte forma: campo (Field): aquilo que está acontecendo, aquilo que o discurso efetivamente é; relações (Tenor): quem está participando da interação, a relação estabelecida entre eles; modo (Mode): qual é papel da linguagem. Tal modelo coloca o aspecto social da linguagem como parte integrante de sua constituição. Por não ser monossistêmica, a variação, em sua relação com o contexto, é algo que precisa ser levado em conta, visto que as escolhas podem estar em relação simbiótica com a situação. O registro teria um importante papel não apenas na produção de textos, mas também em sua compreensão, uma vez que aquilo que buscamos comunicar certamente será 
interpretado com base em nosso conhecimento (MATTHIESSEN, 1993).

Por conseguinte, o registro seria responsável pela instanciação das escolhas no nível da léxico-gramática, ao passo que o contexto de cultura estaria relacionado à esfera social (MARTIN, 1992). Como resultado, o registro é primordial não só para a realização da linguagem, como também para que os elementos específicos da situação de realização se relacionem. Para Eggins (2004), o contexto de cultura é necessariamente mais abstrato que o de situação por um simples motivo: o primeiro tem sua instanciação obrigatoriamente mediada pelo segundo. Em outras palavras, se, por um lado, o contexto de cultura é abstrato e traduz de forma difusa nossas convenções sociais, o contexto de situação é responsável por materializar essas convenções em escolhas linguísticas tangíveis para os falantes.

Uma vez que a língua é um sistema semântico-funcional, as escolhas condicionadas por essas metafunções refletem questões estruturais além de ideológicas.

O sistema de avaliatividade se desenvolve a partir da LSF e tem em seu bojo a análise de como avaliamos, engajamo-nos e intensificamos as diferentes instâncias de participação discursiva. O modelo proposto por Martin e White (2005) é composto por três outros subsistemas: atitude, engajamento e gradação. A atitude poderia ser definida como a expressão de sentimentos. Ela pode abranger três subtipos: o afeto, expressando sentimentos positivos ou negativos, o julgamento, relacionado às atitudes e comportamentos humanos que avaliamos, e a apreciação, que possui caráter estético. O sistema de engajamento está ligado às diferentes possibilidades de posicionamento das diferentes vozes nos textos. Aqui o conceito de heteroglossia é responsável por nos ajudar a compreender a forma como o autor demonstra seu alinhamento em relação às diferentes vozes do texto.

O sistema de gradação, foco deste estudo, tem por propósito intensificar ou mitigar nossas avaliações, podendo, por vezes, ser também um indicativo discursivo de engajamento. Destarte, esse sistema instancia uma avaliação em forma de escala, que pode se desenvolver em dois eixos: força, relacionada à intensidade, e foco, que processa significados ligados à precisão e à prototipia (MARTIN; WHITE, 2005).

$\mathrm{O}$ foco traz à baila categorias que exprimem um sistema classificatório que define unidades de escala que podem não ser sistemas de grandeza perfeitos. Isso levaria tanto à possibilidade de criação de taxonomias bem formadas como de escalas que trabalham por similaridade (MARTIN; WHITE, 2005). No caso de escalas indefinidas, em língua portuguesa, são possíveis comparações, 'Isso é um tipo de carne' ou 'É mais ou menos parecido', responsáveis por criar relações de abrandamento, ao passo que escalas definidas poderiam se definir por adjetivos que acabariam por acrescentar variações por precisão, 'Isso 
que pode até ser um churrasco!'. Os significados de prototipia, por seu turno, também trazem relações escalares que podem estar presentes em epítetos, 'Este é um churrasco de verdade!'. O campo semântico de foco também pode ser considerado atitudinal, já que, ao utilizarmos significados de precisão ou de abrandamento, estamos necessariamente expressando uma perspectiva subjetiva em relação à natureza de uma determinada entidade. Por conseguinte, a questão do foco pode ser representada por uma metáfora visual: o zoom de uma lente objetiva que aproxima o olhar de características específicas deste objeto.

A força, por sua vez, está relacionada a avaliações relacionadas à intensidade e à quantidade. Elas podem operar modificando atributos, 'Esse livro é muito chato', processos, 'Nossa, eu rio muito!', ou elementos modais, ‘É bem possível!’. A intensidade é a avaliação em termos do grau de incremento ou enfraquecimento de uma qualidade, 'ele é bastante bonito' ou 'ele é um pouco bonito', ao passo que a quantidade está relacionada com a medida subjetiva que aplicamos a um substantivo, 'Ele tem pouca moral' (VIAN JR; LIMA-LOPES, 2005).

Como já colocamos anteriormente, há diversos estudos que discutem a questão da avaliatividade em Língua Portuguesa. Entretanto, são poucos os que, como Vian Jr. (2009), discutem estratégias lexicais e morfológicas de gradação, com especial atenção para os processos de sufixação, modificação nominal e processos. Desta forma, a relevância deste trabalho está em discutir estratégias lexicais de gradação a partir de um corpus de interações realizadas em um espaço de comunicação tecnologicamente mediada, como é o caso dos comentários do YouTube, a partir de uma metodologia quali-quantitativa.

\section{Metodologia}

Além da LSF, esta pesquisa conta com os fundamentos teórico-metodológicos e ferramentas da Linguística do Corpus (BAKER, 2009; BONELLI, 2012). Essa escolha foi feita porque essa abordagem torna possível trabalhar com grandes quantidades de textos de forma mais eficiente. Segundo Lima-Lopes (2017), baseado nos trabalhos de Hasan (2014) e Stubbs (1996), a possibilidade de combinação dessas abordagens reside em sua ancoragem comum na Linguística neo-firthiana, uma vez que ambas partem da análise de dados coletados em situações reais de comunicação de forma a buscar padrões de instanciação significativos, além partirem da indissociabilidade entre forma e significado. É importante ressaltar que esta pesquisa se enquadra em um campo metodológico chamado métodos mistos, que se caracterizaram por uma abordagem que considera perspectivas diversas nos processos de 
análise científica (JOHNSON et al., 2007). Apesar de a ideia de coadunar métodos qualitativos e quantitativos não ser algo novo, é importante observarmos que, contemporaneamente, tal movimento surge como uma revisão dos processos dicotômicos previamente estabelecidos entre essas duas abordagens.

De acordo com o próprio site do YouTube (2020), trata-se de uma plataforma de compartilhamento de vídeos criada em 2005, tendo se tornado uma subsidiária da Google em 2006. Seu primeiro vídeo, "Me at the zoo", foi publicado em abril de 2005 por um dos fundadores da plataforma, Jawed Karim. Atualmente, ele é organizado em canais e perfis que disponibilizam vídeos de forma gratuita ou sob assinatura, contando com mais de dois bilhões de usuários. A plataforma possui versões específicas em 100 países, incluindo o Brasil, e já publicou mais de 800 milhões de vídeos.

Neste artigo, analisamos os comentários em resposta ao vídeo "TOP 10 MELHORES TIRADAS DE JAIR BOLSONARO” (KEVINTDFW, 2018), publicado em 7 de outubro de 2018 na plataforma YouTube, e que conta, hoje, com mais de 6.900 .000 visualizações. O produtor de conteúdo por trás do material apresenta seu canal, que hoje tem mais de 1,7 milhão de inscritos e 280 milhões de visualizações, como: "Aqui você encontra patadas uma atrás da outra, tiradas, Thug Life's, e de vez em quando entretenimento em geral" (KEVINTDFW, 2018). Os vídeos postados seguem o mesmo estilo: uma figura pública é selecionada e, com a compilação de frases consideradas pelo produtor de conteúdo como "patadas" ou "tiradas", demonstra sua superioridade em relação a um "rival". No caso do vídeo analisado, a figura pública de superioridade é Jair Bolsonaro e seus "rivais" são, em sua grande maioria, jornalistas.

Os dados desta pesquisa são compostos por comentários extraídos do vídeo "TOP 10 MELHORES TIRADAS DE JAIR BOLSONARO” (ver descrição anterior) por meio de um sistema de raspagem de dados automática baseado na plataforma $\mathrm{R},{ }^{1}$ que também foi utilizada na análise. $\mathrm{R}$ foi inicialmente criado para análise estatística e tratamento de dados (estruturados e não estruturados), mas, nos últimos anos, tem tido significativa presença em estudos da linguagem, especialmente por sua flexibilidade e possibilidade de integração com outras plataformas de pesquisa, como Python, SPSS entre outras.

A tabela 1 traz a descrição do corpus. Como podemos observar, são 5133 comentários, sendo que há um total de 69228 formas (palavras totais) e 55125 tipos (palavras únicas) com uma razão de 0.07. A razão entre os tipos e formas é uma medida universal no estudo de

\footnotetext{
${ }^{1}$ Disponível em: https://www.r-project.org/. Acesso em: 1 fev. 2020. 
corpus e tenta representar um espaço entre 0 (repetição infinita de uma palavra) e 1 (não repetição de palavras); sendo que números como os expressos neste corpus (0.07) apresentam um baixo índice de criatividade e repetições constantes (BIBER; CONRAD; LEECH, 2011). Esse é um resultado já observado no estudo de outras mídias sociais (LIMA-LOPES, 2018) e sua explicação parece estar no senso de comunidade e pertencimento trazido pela repetição lexical. Os comentários não são extensos, possuindo, em média, de 13.49 formas, 10.74 tipos e 64.69 caracteres.

\begin{tabular}{l|c} 
& VALORES \\
\hline COMENTÁRIOS & 5133 \\
TOTAL DE TIPOS & 55125 \\
TOTAL FORMAS & 69228 \\
MÉDIA TIPOS & 10.74 \\
MÉDIA FORMAS & 13.49 \\
MÉDIA DE CARACTERES & 64.69 \\
RAZÃO TIPOS/FORMAS & 0.07
\end{tabular}

Tabela 1: Descrição do Corpus

Fonte: elaborada pelo autor

Neste artigo, de forma a realizar a coleta e análise de dados, os seguintes pacotes foram utilizados: 1) TubeR (SOOD, 2020): capaz de interagir com a API (Application Programming Interface) do YouTube e realizar o download dos comentários dos vídeos. A aquisição dos comentários exige que o pesquisador possua uma conta de desenvolvedor, obtida junto ao Google ${ }^{2}$ 2) Tidyverse (WICKHAM et al., 2019): permite a manipulação de tabelas e grupos de dados, assim como a separação das expressões regulares; 3) Quanteda (BENOIT et al., 2018): permite a criação de matrizes textuais, facilitando a pesquisa por meio de concordâncias e listas de trigramas; 3) Corrplot (WEI; SIMKO, 2017) e Ggcorrplot (KASSAMBARA, 2020): permitem representações visuais dos cálculos de correlação.

Após a raspagem dos comentários de forma automática, foi realizada uma lista geral de palavras, de forma a observar as ocorrências da palavra mito e classificar quais estratégias estariam presentes em cada uma delas. O levantamento das estratégias, a frequência de cada uma foi computada por instância de ocorrência no corpus de estudo. Para essa fase de classificação, utilizamos uma planilha tradicional de dados. É importante ressaltar que a fase de classificação foi realizada de forma manual, partindo das de concordâncias e da lista geral de palavras, observando o total de ocorrências. Uma descrição detalhada dessas categorias é

\footnotetext{
${ }^{2}$ Disponível em: https://developers.google.com. Acesso em: 1 fev. 2020 
apresentada na seção a seguir.

Tais dados foram incorporados aos scripts em R e normalizados por 1000, de forma a proporcionar a amostra antes da aplicação do teste de correlação, nesse caso R-Pearson (BREZINA, 2018). A função desse teste é observar quais estratégias tendem (ou não) a coocorrer em uma escala que varia entre -1 e 1 . Em outras palavras, cada vez que duas estratégias possuem um score próximo a 1, elas tendem a estar juntas nos comentários, ao passo que números próximos a -1 indicam o contrário. O valor zero, por sua vez, implica que não há correlação na ocorrência, sendo, aqui, interpretado como casos de aleatoriedade. Todos os scripts escritos para este estudo estão disponíveis para download no sítio dos pesquisadores. ${ }^{3}$ As unidades de análise utilizadas nesta pesquisa emergiram exclusivamente da observação subjetiva dos dados. Como colocado na seção anterior, partimos de uma lista de palavras nas quais as unidades foram definidas com o auxílio de um pacote em $\mathrm{R}$ para realização de concordâncias. Tais unidades caracterizam-se por uma série de estratégias de intensificação, aplicadas aos comentários selecionados. De maneira geral, foram observadas as estratégias constantes no quadro 1.

\begin{tabular}{l|l|l}
\multicolumn{1}{c}{ Estratégia } & \multicolumn{1}{c}{ Definição } & \multicolumn{1}{c}{ Exemplo } \\
\hline CA: Caixa Alta & Utilização exclusiva de letras maiúsculas & BOLSOMITO \\
\hline CB: Caixa Baixa & Utilizção exclusiva de letras minúsculas & mito \\
\hline SU: Justaposição & $\begin{array}{l}\text { Utilizção da palavra mito na posição de justaposição com } \\
\text { caixa normal }\end{array}$ & Bolsomito \\
\hline CAS: Caixa Alta Sufixo & $\begin{array}{l}\text { Utilização da palavra mito na posição de sufixo com caixa alta } \\
\text { no sufixo }\end{array}$ & bolsoMITO \\
\hline RV: Repetição Vogal & Repetição de uma ou mais vogais da palavra mito & bolsomiiitooo \\
\hline V: Verbo & Neologismo verbal incluindo a palavra mito & Mitou \\
\hline AB: Abreviação & $\begin{array}{l}\text { Abreviação do nome do candidato na construção, } \\
\text { acompanhando a palavra mito }\end{array}$ & Bolmito \\
\hline ANO: ano & Palavra mito composta com o ano da inscrição & bolsomito2018 \\
\hline CC: Letra Camelo & Alternância entre maiúscula e minúscula & JairMito \\
\hline ADJ: Adjetivação & Criação de adjetivos a partir da palavra mito & Mitoso \\
\hline R: Repetição & Repetição da palavra mito sequencialmente no comentário & mito mito mito (...) \\
\hline RS: Repetição Sílaba & $\begin{array}{l}\text { Repetição de uma ou mais sílabas no processo de constituição } \\
\text { da palavra }\end{array}$ & MITOTOTO \\
\hline CXN: Caixa normal & $\begin{array}{l}\text { Uso da palavra mito com o arranjo de maiúsculas e minúsculas } \\
\text { esperado no âmbito da sentença em português Brasileiro }\end{array}$ & Mito \\
\hline D: Demais & $\begin{array}{l}\text { Estratégias com ocorrência muito baixa para computação como } \\
\text { uma categoria }\end{array}$ & $\begin{array}{l}\text { MitoPSL17, } \\
\text { vamobolsomito }\end{array}$ \\
\hline
\end{tabular}

Quadro 1: Estratégias mapeadas

Fonte: Dados de pesquisa

Exemplos em contexto são apresentados durante a análise dos resultados.

\footnotetext{
${ }^{3}$ Os scripts estão disponíveis em http://www.iel.unicamp.br/rll307 e https://github.com/rll307. 


\section{Resultados}

Um levantamento inicial exploratório de forma a observar quais seriam os itens lexicais mais comuns dentro do corpus. A figura 1 traz os resultados de tal análise preliminar, mostrando em qual porcentagem dos documentos as 30 palavras mais frequentes ocorrem. Podemos observar que a palavra mito, sozinha, sem a combinação com outros elementos lexicais, está presente em $71 \%$ dos comentários, ao passo que as demais ocorrem em números inferiores a $1 \%$ cada. A disparidade desses números torna impossível uma representação gráfica proporcionalmente adequada que possibilite uma clara leitura dos números no eixo y, razão pela qual tais números foram acrescentados em forma de legendas sobre o eixo x. Esses resultados são relevantes, pois mostram a importância da palavra mito para a construção do discurso nesse contexto. Foi justamente tal importância que nos levou a analisar mais detalhadamente suas ocorrências no corpus. Além de um simples apelido de campanha, ela parece ser chave na compreensão de como o discurso dos apoiadores do candidato se colocam.

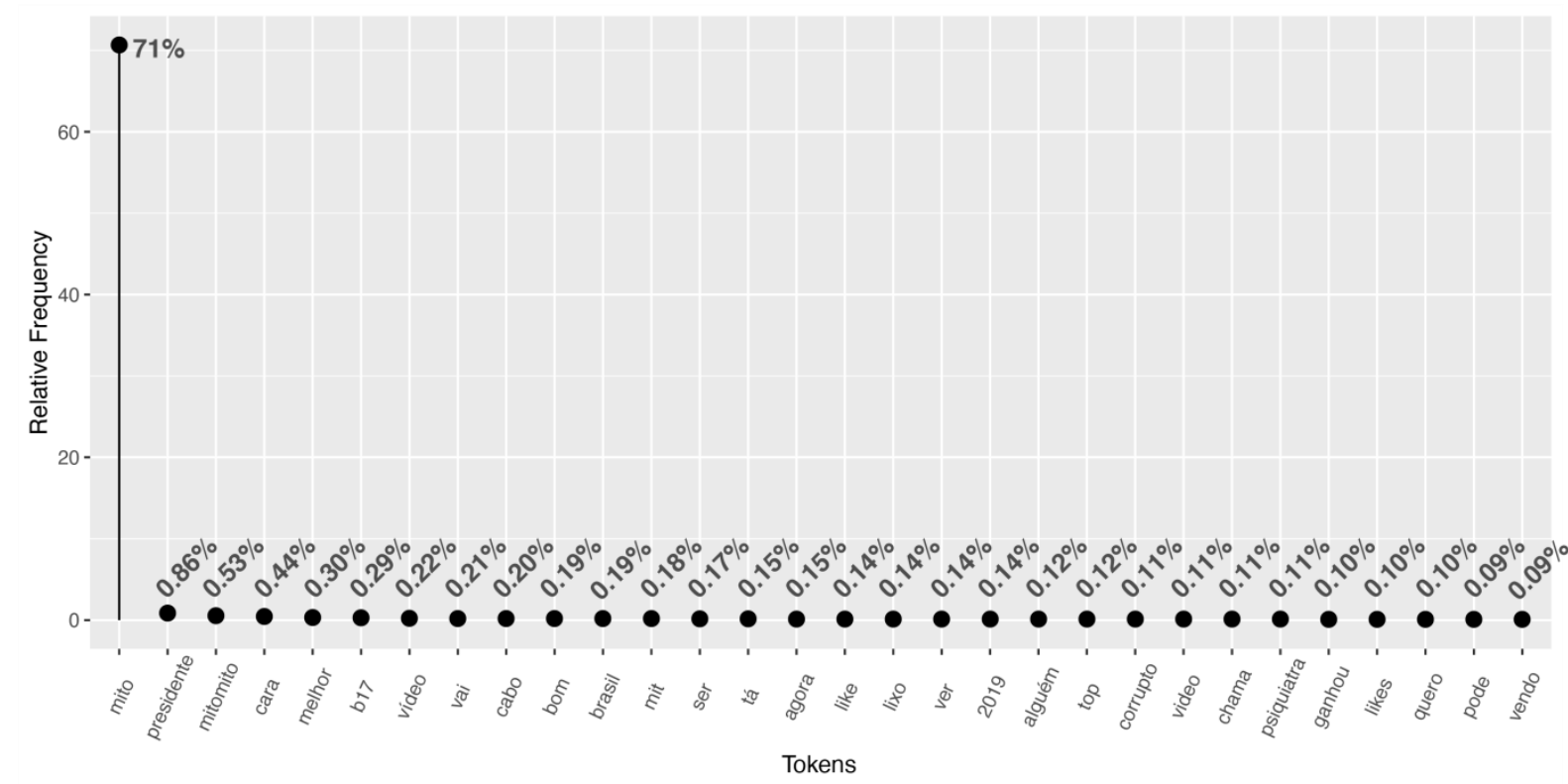

Figura 1: Palavras mais frequentemente distribuídas pelos comentários $(n=30)$ / normalizado por 100

Fonte: Dados de pesquisa

Mito e cara chamam a atenção por serem palavras que definem uma visão afetiva do candidato. No caso da primeira, ela corresponde a um apelido comumente atribuído a ele 
durante a eleição (Ele é o CARA !!!!!), ao passo que a segunda o define como um indivíduo comum, na maioria dos casos em uma expressão idiomática que o identifica positivamente ([...] vim dizer que o Mito é presidente!!).

Há duas formas comuns de intensificação: a primeira está relacionada à sequência de pontos de exclamação; e a segunda, à utilização de letras maiúsculas. Comuns no ambiente digital, tais estratégias podem ocorrer de forma associada (Jair Bolsonaro para presidente 2018.), criando um acúmulo intensificador. Não raro, ambas parecem ocorrer em contextos nos quais há processos relacionais atributivos. Tais processos têm a função de relacionar uma entidade a uma característica a ele combinada, levando a um efeito discursivo de associação. No caso específico dos exemplos do Quadro 1, essa associação tende a ocorrer em proposições também intensificadoras, levando a um empilhamento dessas estratégias.

Os resultados mostram que o uso da palavra mito apresenta uma série de variações ortográficas, responsáveis por diferentes tipos de ênfase, especialmente na perspectiva da intensificação (Figura 2).

Entre as estratégias levantadas, a mais frequente é o uso de caixa baixa (23.12\%) (CB, letras minúsculas), seguida pelo uso esperado da relação maiúscula e minúscula (18.63\%) (CXN: Caixa normal) no corpo do período, ao passo que a abreviatura do nome do candidato (10.66\%) (AB: Abreviação) é a terceira mais frequente. A quarta estratégia em quantidade é a utilização da palavra mito justaposta ao nome do candidato (10.45\%) (SU: Justaposição), que possui número bem próximos à utilização de letras maiúsculas (10.24\%) (CA: Caixa Alta). A repetição de vogais (9.36\%) (RV: Repetição de Vogais) tem uma grande diferença em relação às demais estratégias menos frequentes. Entre elas, estão a repetição de palavras $(5.39 \%)$ (R: Repetição) e a utilização da palavra mito como sufixo em caixa baixa (4.91\%) (CBS: Caixa Baixa Sufixo), e o ano da eleição (2.48\%) (ANO) colocado de forma anexa ao nome do candidato. As demais estratégias possuem números inferiores a $1.5 \%$. Entre elas, estão o uso de Letras Camelo (1.4\%) (CC: Letra Camelo), estratégias diversas (0.98\%) (D: Demais), a utilização de verbo originado da palavra mito $(0.72 \%)$ (V: Verbo), a repetição de sílabas (0.67\%) (RS: Repetição Sílaba) e o uso de derivação adjetival de mito (0.5\%) (ADJ: Adjetivação). Todas essas estratégias são discutidas a seguir. 


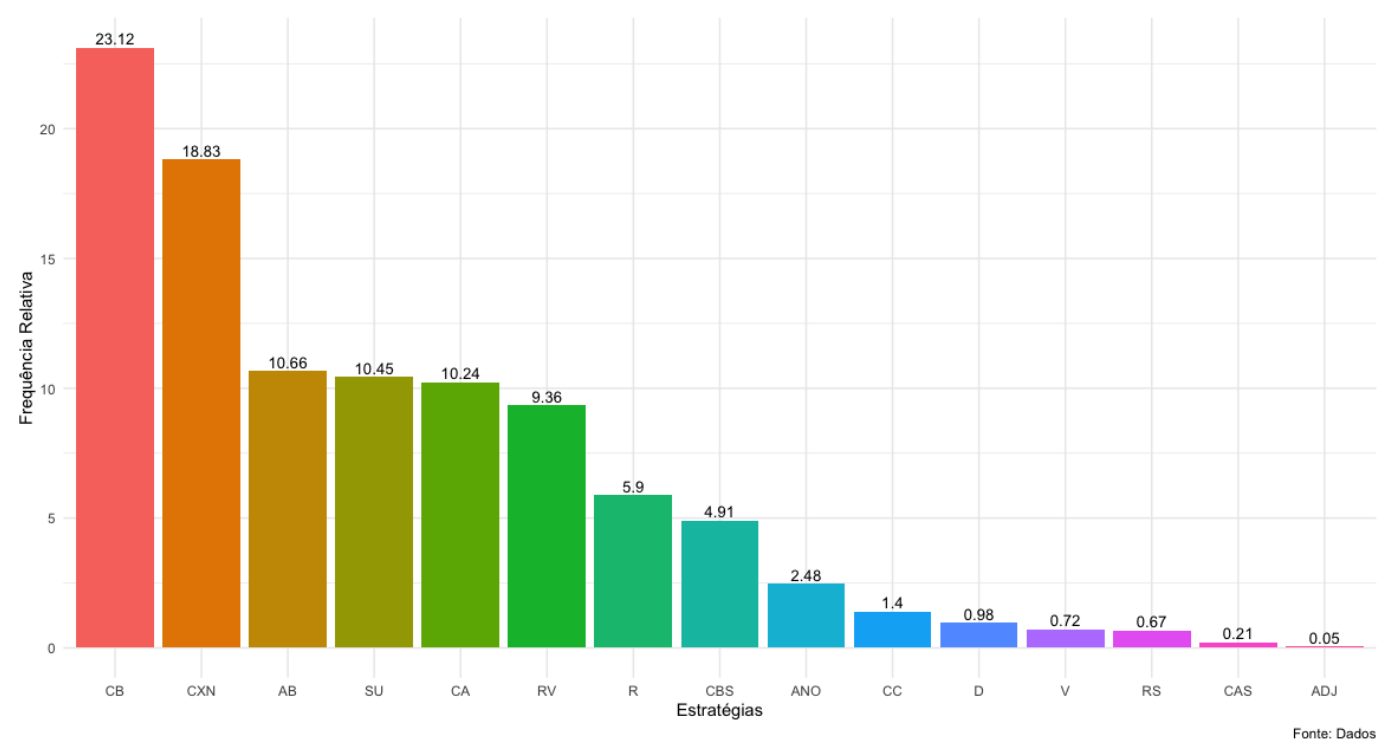

Figura 2: Estratégias de uso da palavra mito

Convenção: CA: Caixa Alta, CB: Caixa Baixa, SU: Justaposição, CAS: Caixa Alta Sufixo, CBS: Caixa Baixa Sufixo; RV: Repetição Vogal, V: Verbo, AB: Abreviação, ANO: Ano, CC: Letra Camelo, D:

Demais, ADJ: Adjetivação, R: Repetição, CXN: Caixa normal, RS: Repetição Sílaba.

Fonte: Dados de pesquisa

A caixa baixa (CB: letras minúsculas), estratégia na qual o uso de letras minúsculas corre independentemente do local na frase em que a palavra é utilizada, está associada à repetição (R) de palavras (linha 3, Quadro 2). Ela, muitas vezes, compõe o único texto do comentário. O uso normal de minúsculas e maiúsculas (CXN: Caixa normal), ou seja, respeitando o uso maiúsculo no início de um período e o minúsculo durante o período, é a segunda estratégia mais comum. No corpus em estudo ela está associada a processos relacionais que caracterizam o candidato de forma positiva (linha 1, Quadro 2), à intensificação pelo uso de advérbios (linha 2, Quadro 2) ou à repetição de vogais (RV) (linha 4, Quadro 2).

\begin{tabular}{|r|r|l|l|}
\hline 1 & Ai é o & mito & bolsonaro \\
\hline 2 & O cara é & mito & mesmo \\
\hline 3 & Esse cara tem meu respeito & mito & mito mito \\
\hline 4 & mito & mito & mitooooo \\
\hline
\end{tabular}

Quadro 2: Concordâncias

Fonte: Dados do corpus

A palavra mito é constantemente utilizada de forma a se justapor (SU: justaposição) o nome abreviado do candidato (AB: Abreviação), fato que justifica suas ocorrências tão próximas (ver Figura 2). A relação entre sufixação ocorre tanto em situações nas quais o 
sufixo ocorre em letra minúscula como em letra maiúscula. Entre as combinatórias possíveis estão: o nome do candidato abreviado com o sufixo em letra minúscula (CBS - Caixa Baixa Sufixo, linha 1, Quadro 3) ou em letra maiúscula (CAS - Caixa Alta Sufixo, linha 2, Quadro 3). Em alguns casos, a variação entre caixa alta e baixa gera um efeito informalmente chamado na área de informática de CamelCase (ou CC - Letras Camelo), que poderia ser definido como a diferenciação de duas palavras escritas sem espaço (linha 3, Quadro 3). Por vezes, esses processos de sufixação podem coocorrer com o ano da eleição, formando uma hashtag que propicia o engajamento e indexação dos comentários (linha 4, Quadro 3).

\begin{tabular}{|l|r|c|l|}
\hline 1 & nos brasileiro já acostumamos com esses lixo do q tá aí, mas & bolsomito & vem aí \\
\hline 2 & $(\ldots)$ & Bolsomito & $\begin{array}{l}\text { 2019 já esse é o nosso } \\
\text { presidente }\end{array}$ \\
\hline 3 & & \#BolsoMito & \\
\hline 4 & & \#BolsoMito2018 & \\
\hline
\end{tabular}

Quadro 3: Concordâncias

Fonte: Dados do corpus

O termo mito passa a ser utilizado como um verbo (linha 1, Quadro 4) e está constantemente associado ao uso de emoticons, como demonstração de avaliação de afeto. Outro ponto de transformação está na criação de adjetivos para a caracterização do candidato, como é o caso de mitoso (linha 2, Quadro 4), algo também relacionado à sua performance. Os processos de repetição podem se manifestar de duas formas: na primeira, e mais comum, há a repetição de vogais (RV), que podem ocorrer tanto na vogal final de um substantivo (linha 3, Quadro 4) como na sílaba final de um verbo (linha 5, Quadro 4) ou em todas as vogais do substantivo (linha 6, Quadro 4). Em muitos casos, essa repetição pode ocorrer dentro de um período (linha 4, Quadro 4), ou ocorrer sozinha, formando um comentário exclusivamente por uma palavra ou hashtag (linha 6, Quadro 4).

\begin{tabular}{|r|r|r|l|}
\hline 1 & Dá que eu te dou outra kkkkkkk & mitou & ? \\
\hline 2 & Kkk & mitoso & 2018 e nosso ano \\
\hline 3 & Mito mito mitoto & mitoto & \\
\hline 4 & tira o microfone não & MITOOOOOOO & \\
\hline 5 & E NOSSAAAAAA, & MITOOOOOOOUUUUUUUUUU & \\
\hline 6 & & Miiiiiiitoooooooo & \\
\hline 7 & MITO , & MITO & , MITO, MITO \#BOLSONARO2022 \\
\hline
\end{tabular}

Quadro 4: Concordâncias

Fonte: Dados do corpus

Como também é possível observar, a repetição de sílabas ou letras pode estar 
associada a uma série de estratégias. Um exemplo é a utilização de letras minúsculas no início da sentença (linha 4, Quadro 4), assumindo-se que não seja um termo da oração, mas sim uma marca paralinguística para indicação de risadas. O segundo é a repetição de palavras (R), na qual um termo é constantemente repetido dentro do post (linha 7, Quadro 4), ora em caixa normal (CXN), a exemplo da linha 3 (Quadro 4), ora em letras maiúsculas (CA: Caixa Alta) como nas linhas 5 e 7 (Quadro 4).

As demais estratégias (D: Demais) são formadas por um grupo de usos que não se repetem, ocorrendo exclusivamente em um comentário. Entre elas, estão a utilização do primeiro nome do candidato em uma configuração de letras camelo (linha 1, Quadro 5), das iniciais do candidato em letra maiúscula (CA - Caixa Alta, linha 2, Quadro 5), ambas justapostas à palavra mito, e da utilização de número e sigla de seu partido (linha 3, Quadro $5)$.

\begin{tabular}{|c|l|c|l|}
\hline 1 & $\begin{array}{l}\text { concerteza o Jair bolsonaro vai mudar de vez esse Brasil } \\
\text { corrupto . }\end{array}$ & \#JairMito & $\begin{array}{l}\text { \#FUTUROpresidente } \\
\text { \#Brasilmelhor }\end{array}$ \\
\hline 2 & Só eu que vi a mulher rindo da oprimida do & JBMito & na Repórter \\
\hline 3 & Corte rápido, faca é TRAMONTINA ! ! KK & \#MitoPSL17 & \\
\hline
\end{tabular}

Quadro 5: Concordâncias

Fonte: Dados do corpus

Tais escolhas têm impacto na forma como o escritor coloca sua ênfase no comentário, uma vez que a palavra mito adquire diferentes configurações graças às estratégias utilizadas. De acordo com Martin \& White (2005), as escolhas de intensificação podem sofrer um processo de 'empilhamento'. Na análise realizada pelos autores, isso ocorreria, especialmente, pela atribuição contínua de características ou uso de advérbios de intensificação justapostos. No caso específico dos dados desta pesquisa, tal estratégia parece não ocorrer. Isso, porque a natureza do vídeo que é de enaltecimento ao candidato, como discutimos na seção de metodologia, parece dirigir a interação para um caminho específico.

Assim, se nos voltarmos para os exemplos presentes nos Quadros 1 e 5, observaremos que os comentários parecem mostrar que a interação ganha um caráter de torcida a favor do candidato e de sua vitória. Eles são repetitivos à medida que parecem apenas reiterar palavras de ordem e de apoio. Em outros termos, os usuários do YouTube não estariam interessados em discutir aquilo que aparece no vídeo, apenas fazem comentários de forma a reforçar seu pertencimento a essa comunidade. Tais resultados corroboram a discussão proposta por Sovignon \& Roithmeier (2013) e Lima-Lopes (2018). Os primeiros, ao analisarem a interação entre estudantes de línguas estrangeiras em um Bolletin Board, concluem que parte relevante 
da noção de pertencimento a uma comunidade está na reutilização de trechos já postados, ou na forma de reaproveitamento de trechos ou no uso de uma estrutura linguística comum (SOVIGNON; ROITHMEIER, 2013). Já Lima-Lopes (2018) observa que diversos usuários, durante um protesto conservador no Facebook, tendem a copiar uma mesma mensagem e a repostá-la. Tal mensagem foi, inicialmente, publicada por um usuário influente dentro da rede de conservadores que se opunha a uma exposição de artes.

Essa forma de pertencimento que se manifesta pela repetição de ideias e de escolhas lexicais talvez possa explicar por que, em diversos casos, algumas escolhas congregam mais de uma estratégia de ênfase. Um exemplo seria Bolsomito (linha 2, Quadro 3), que instancia uma palavra composta pelo sobrenome do candidato justaposta à palavra mito, ou \#JairMito (linha 1, Quadro 5), que utiliza uma estratégia similar, mas emprega o primeiro nome do candidato e alterna letras maiúsculas e minúsculas (ou letra camelo). Outros exemplos seriam MITOOOOOOOUUUUUUUUUU (linha 5, Quadro 4), que transforma o substantivo mito em um verbo, além de repetir as vogais finais, simulando o prolongamento da vogal em escolha fonológica claramente avaliativa, e a caixa alta, simulando o grito. A repetição da palavra mito (linha 3, Quadro 4) também é uma estratégia que, presente nos dados, busca intensificar a avaliação positiva do candidato.

Assim, o propósito comunicativo (HALLIDAY; HASAN, 1991) dos comentários parece ser reafirmar o pertencimento à comunidade de apoiadores do presidente por meio de avaliações positivas do candidato, intensificadas (MARTIN; WHITE, 2005) por diferentes escolhas (HALLIDAY, 1978) envolvendo a palavra mito e/ou o nome do candidato. Tal observação nos leva a questionar se tais escolhas ocorrem de forma aleatória ou se haveria alguma correlação entre elas. Uma possível correlação poderia levar ao estabelecimento de padrões mapeáveis (Figura 4). 


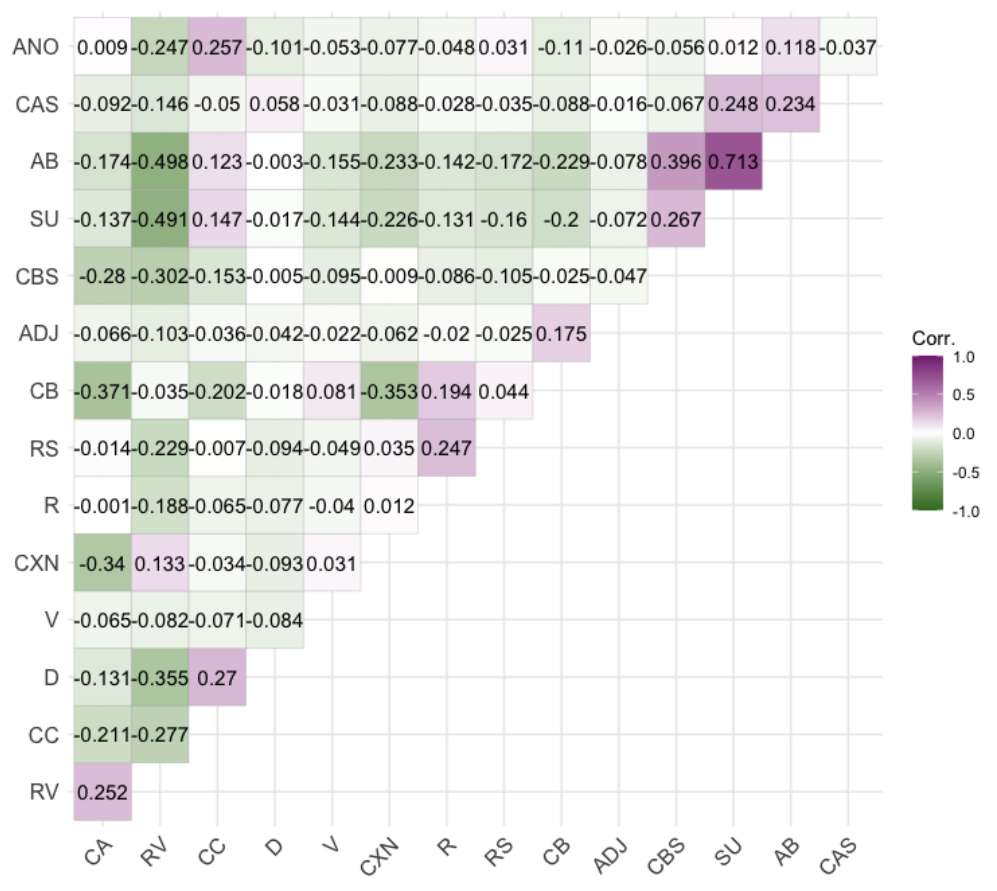

Figura 4: Correlação entre estratégias

Convenção: CA: Caixa Alta, CB: Caixa Baixa, SU: Sufixação, CAS: Caixa Alta Sufixo, CBS: Caixa

Baixa Sufixo; SU: Justaposição, RV: Repetição Vogal, V: Verbo, AB: Abreviação, ANO: Ano, CC:

Letra Camelo, D: Demais, ADJ: Adjetivação, R: Repetição, CXN: Caixa normal, RS: Repetição

Sílaba.

Fonte: Dados de pesquisa

A Figura 4 traz o teste de correlação aplicado às estratégias de intensificação relacionadas à palavra mito. As cores e os números representam o grau e a natureza da correlação entre as estratégias: os tons arroxeados implicam a correlação positiva e os esverdeados a negativa, ao passo que o valor da correlação é representado por números - tons mais escuros implicam uma correlação mais forte (seja negativa ou positiva). Como já discutimos na seção de Metodologia, a escala pode variar entre -1 (correlação negativa) e 1 (correlação positiva), sendo que o zero (cor branca) implica a não existência de correlação. Neste estudo, a correlação positiva implica a probabilidade de as duas estratégias formarem um padrão de coocorrência nos textos analisados; já a negativa implica a tendência de as estratégias não coocorrerem, formando um padrão de autoexclusão.

Entre as correlações positivas mais representativas estão a utilização da palavra mito justaposta (SU) e abreviação do nome do candidato (AB). A abreviação $A B$ também está relacionada positivamente à utilização da palavra mito em caixa baixa durante o processo de construção da palavra (CBS), além das letras camelo (CC). Correlação positiva também foi apresentada entre $\mathrm{AB}$ e a palavra mito justaposta em caixa alta (CAS) e a abreviatura $(\mathrm{AB})$. $\mathrm{A}$ 
repetição de palavras $(\mathrm{R})$ está positivamente relacionada à repetição de sílabas e à utilização de palavras em caixa baixa (CB).

A escolha de letras maiúsculas, ou caixa alta (CA), está positivamente relacionada com a repetição de vogais em sílabas finais (RV), ao passo que a utilização do ano da eleição justaposto em relação à palavra mito, ou ao nome do candidato, correlaciona-se positivamente com o uso de letras camelo (CC), essas também frequentemente ocorrendo com as demais estratégias (D) e à justaposição do ano.

Já as correlações negativas, ou seja, estratégias que tendem a não estar juntas no mesmo comentário, também trazem alguns resultados relevantes. A utilização de mito como adjetivo, por exemplo, tende a ter uma fraca relação negativa com quase todas as demais estratégias - à exceção do uso em caixa baixa (CB), ao passo que mito como verbo está negativamente correlacionada a todas as demais estratégias. Entre as correlações mais significativas estariam a repetição de vogais (RV), a justaposição (SU), a abreviação $(A B)$ e a caixa baixa do sufixo (CBS), algumas delas estando entre as principais em termos de ocorrência simples (ver Figura 3).

Se compararmos os resultados das Figuras 3 e 4, observaremos que as estratégias mais frequentes raramente possuem correlação positiva. Se, em um primeiro momento, tal resultado pode significar que elas não necessariamente coocorram, também podem trazer à baila questões relacionadas à natureza dos modos de intensificação. Se, de um lado, haveria estratégias centrais, cuja utilização naturalmente exclui outras igualmente centrais, de outro, estratégias menos comuns, ou ancilares, seriam responsáveis por completar o sentido da intensificação. Tais resultados são importantes por confirmarem um importante construto da LSF, a noção de escolha.

Para Fawcett (2013), as escolhas refletem nossos sistemas de crenças, representando um modelo implícito de língua aplicável a um determinado contexto de situação. Tais escolhas não seriam arbitrárias, mas motivadas pelo contexto e autoexclusivas, à medida que uma escolha necessariamente elimina a possibilidade de coexistência de outra com a mesma função em um determinado texto (THOMPSON, 2004). Fawcett (2013) observa que o conceito de escolha pode ser ampliado de forma a considerar aquelas que "podem" ser realizadas e aquelas que o "devem" ser, enquanto produto potencial de linguagem. Por conseguinte, o usuário parece não estar exclusivamente escolhendo esta ou aquela estratégia de intensificação, mas um sistema que está centrado em algumas estratégias principais que se ramificam em outras possíveis escolhas. 


\section{Considerações finais}

Este artigo teve por objetivo estudar os processos de intensificação em uma seção de comentários extraída de um vídeo de apoio ao candidato à presidência da república Jair Bolsonaro no YouTube e compreender como tais processos ajudam a construir uma imagem positiva da figura pública. O trabalho teve, como base teórica, a Linguística SistêmicoFuncional (HALLIDAY, 1978, 2013) e o sistema de avaliatividade (MARTIN; WHITE, 2005; THOMPSON; ALBA-JUEZ, 2014), com foco no subsistema de gradação. De forma a atingir os objetivos e responder às perguntas propostas, a Linguística do Corpus (BAKER, 2009; BONELLI, 2012) foi a ferramenta escolhida para levantamento, coleta e análise dos 5.133 comentários do vídeo selecionado.

Os comentários mostram um caráter de torcida a favor do candidato à presidência, o que os faz apresentar certos padrões linguísticos e grafológicos, reiterando palavras de ordem e de apoio. Assim, nenhum dos comentários analisados discute o vídeo em questão, mas, sim, demonstram adesão ao candidato através da repetição de ideias e escolhas lexicais, marcando, com isso, sua posição naquela comunidade. Em tais comentários, a escolha que mais se destacou foi mito, devido à sua variação ortográfica, o que aponta para o uso de diversas estratégias de intensificação.

Namise (2019) discute o uso da palavra mito relacionada a Jair Bolsonaro e conclui que ela funciona como uma estratégia digital para conquistar notoriedade, algo definido na linguagem de internet como uma trollagem. Ao chamar Bolsonaro de "mito", seus apoiadores não estão exaltando sua capacidade intelectual, mas, sim, de sua habilidade em causar indignação nos alvos certos. Para seus apoiadores, toda declaração que irrite a oposição é digna de ser considerada uma "mitada" (NAMISE, 2019, p.72). É nesse regime que a utilização das estratégias levantadas neste estudo se coloca, a perspectiva principal não é mais discutir propostas políticas ou programas, mas estabelecer um contexto de situação no qual o impacto discursivo é traduzido em avaliação positiva.

Como coloca Silva (2018), os canais dedicados ao regime de pós-verdade parecem se filiar a um regime discursivo que busca estabelecer relações por meio das contradições e inferências que pretendem construir. Como estamos em uma era caracterizada pelo compartilhamento rápido de vídeos, tuítes e posts, a difusão e circulação em massa desses conteúdos questionáveis através do engajamento dos usuários gera um efeito de verdade, legitimando-os. Sendo assim, os produtores de conteúdos falam para uma audiência que valida tudo o que é dito, criando uma comunidade que é guiada pelo seu sistema de crenças, 
sem a possibilidade de diálogo (BENTES, 2018).

Como o propósito comunicativo (HALLIDAY; HASAN, 1991) dos usuários parece ser o mesmo, analisamos as estratégias de intensificação de forma a entender se há aleatoriedade ou correlação na maneira como estão dispostas, o que poderia levar a padrões mapeáveis. A correlação positiva mais representativa se deu entre a utilização da palavra mito como justaposição e a abreviação do nome do candidato (AB). Ao analisarmos as correlações negativas, percebemos que, enquanto a utilização de mito como adjetivo tem uma fraca relação negativa com as estratégias apresentadas, sua utilização como verbo está negativamente ligada a quase todas elas.

É importante ressaltar que este estudo traz contribuições em, pelo menos, dois campos. O primeiro é o metodológico. Ao observarmos quais são as estratégias e como elas se configuram em termos de correlações, é possível observar que isso não se dá de forma aleatória dentro do contexto dos comentários. Nesse sentido, podemos observar que as estratégias não são livremente combináveis: a escolha pressupõe um pacote de possibilidades que estão relacionadas às combinatórias aceitas pela comunidade.

O segundo está relacionado às estratégias típicas de gradação utilizadas por uma comunidade virtual específica. Compreender esses mecanismos é importante para refletirmos como a adesão a uma comunidade se dá pela linguagem em contextos da Comunicação Mediada por Computador.

\section{Agradecimentos}

Rodrigo Esteves de Lima-Lopes, agradece ao CNPq (processo 422111/2018-0) pelo financiamento. Os autores agradecem a Sara Regina Scotta Cabral (UFSM), a Lucia Rottava (UFRGS) e a Rosinda de Castro Guerra Ramos (PUCSP) pela cuidadosa leitura e revisão.

\section{REFERÊNCIAS}

ALBA-JUEZ, L.; THOMPSON, G. The many faces and phases of evaluation. In: THOMPSON, G.; ALBA-JUEZ, L. (Eds) Evaluation in context. Pragmatics \& Beyond New Series. Amsterdam; Philadelphia: John Benjamins Publishing Company, 2014. p. 3-27.

ALMEIDA, F. A. S. D. P. Atitude: afeto, julgamento e apreciação. In: VIAN JR, O.; SOUZA, A. A. DE; ALMEIDA, F. A. S. D. P. (Eds) A linguagem da avaliação em Língua Portuguesa. São Carlos: Pedro \& João Editores, 2011a. p. 99-112. 
ALMEIDA, F. A. S. D. P. Comentários em blogs de professores de inglês: uma análise do sistema de Avaliatividade. DELTA: Documentação de Estudos em Linguística Teórica e Aplicada, São Paulo, v. 34, n. 1, p. 181-204, mar. 2018.

ALMEIDA, F. A. S. D. P. Os recursos léxico-gramaticais de atitude no discurso de dois professores universitários. In: VIAN JR, O.; SOUZA, A. A. DE; ALMEIDA, F. A. S. D. P. (Eds) A linguagem da avaliação em Língua Portuguesa. São Carlos: Pedro \& João Editores, 2011b. p. 181-196.

ALMEIDA, F. A. S. D. P.; VIAN JR, O. Estudos em avaliatividade no Brasil: panorama 2005-2017. Signótica, Goiânia, v. 30, n. 2, p. 273, 2018.

ARAÚJO, W.; MONTARDO, S. Ciberativismo em sites de redes sociais: Uma análise da apropriação das páginas do Facebook pela WikiLeaks. Redes.com, [s. l.], v. 8, p. 71-84, 2013.

BAKER, P. (Ed.) Contemporary Corpus Linguistics. London: Continuum, 2009.

BEAUGRANDE, R. Register in discourse studies: A concept in search of a theory. In: GHADESSY, M. (Ed) Register Analysis. Theory and Practice. Londres, Nueva York: Pinter Publishers. New York: Pinter Publishers, 1993. p. 7-25.

BENOIT, K. et al. Quanteda: An R package for the quantitative analysis of textual data. Journal of Open Source Software, [s. l.] ,v. 3, n. 30, p. 774, 6 out. 2018.

BENTES, I. Economia Narrativa: Do Midiativismo aos Influenciadores Digitais. In:

BRAIGHI, A. A.; LESSA, C. H.; CÂMARA, M. T. (Orgs.) Interfaces do Midiativismo: Do conceito à prática. Belo Horizonte: CEFET-MG, 2018. p. 111-131.

BREZINA, V. Statistics in corpus linguistics: a practical guide. Cambridge; New York: Cambridge University Press, 2018.

BERBER-SARDINHA, T. Linguística de Corpus. São Paulo: Manole, 2004.

BONELLI, E. T. Theoretical overview of the evolution of corpus linguistics. In: O'KEEFFE, A.; MCCARTHY, M. (Eds.) The Routledge handbook of corpus linguistics. Routledge Handbooks in Applied Linguistics. New York: Routledge, 2012. p. 14-28.

BIBER, D.; CONRAD, S.; LEECH, G. N. Longman student grammar of spoken and written English.Harlow: Longman, 2011.

BRAIGHI, A. A.; CÂMARA, M. T. O que é midiativismo? Uma proposta conceitual. In: BRAIGHI, A. A.; LESSA, C.; CÂMARA, M. T. (Eds) Interfaces do Midiativismo: do conceito à prática. Belo Horizonte: CEFET-MG, 2018. p. 25-42.

CABRAL, S. R. S. A mídia e o presidente: um julgamento com base na teoria da valoração. 2007. 247 f.. Tese (Doutorado em Letras) - Universidade Federal de Santa Maria, Santa Maria, 2007.

CABRAL, S. R. S. Estima ou sanção: onde está a opinião? In: VIAN JR, O.; SOUZA, A. A. 
DE; ALMEIDA, F. A. S. D. P. (Eds) A linguagem da avaliação em Língua Portuguesa. São Carlos: Pedro \& João Editores, 2011. p. 151-166.

CASTELLS, M. Networks of outrage and hope: social movements in the Internet age. Cambridge, UK ; Malden, MA: Polity Press, 2015.

CRYSTAL, D. Towards a typographical linguistics. Linguistics, [s. l.], v. 17, p. 7-23, 1997.

EGGINS, S. An introduction to systemic functional linguistics. $2^{\text {nd }}$ ed. New York: Continuum, 2004.

EGGINS, S.; MARTIN, J. R. Genres and Registers of Discourse. In: VAN DIJK, T. A. (Ed.) Discourse as Structure and Process: Discourse Studies: A Multidisciplinary Introduction. London: SAGE Publications Ltd, 1997. p. 230-256.

FALLIS, D. What Is Disinformation? Library Trends, [s. l.], v. 63, n. 3, p. 401-426, 2015.

FAWCETT, R. P. Choice and choosing in Systemic Functional Grammar: What is it and how is it done? In: FONTAINE, L.; BARTLETT, T.; OGRADY, G. (Eds.) Systemic Functional Linguistics: Exploring Choice. Cambridge: Cambridge University Press, 2013. p. 115-134.

GABARDO, M.; LIMA-LOPES, R. E. DE. Ni una menos: ciência das redes e análise de um coletivo feminista. Humanidades \& Inovação, [s. l.], v. 5, n. 3, p. 44-58, 2018.

HALLIDAY, M. A. K. Language as social semiotic: the social interpretation of language and meaning. London: University Park Press, 1978.

HALLIDAY, M. A. K. Meaning as choice. In: FONTAINE, L.; BARTLETT, T.; O'GRADY, G. (Eds) Systemic Functional Linguistics: Exploring Choice. Cambridge: Cambridge University Press, 2013. p. 15-36.

HALLIDAY, M. A. K.; MATTHIESSEN, C. M. I. M. Halliday's introduction to functional grammar. $4^{\text {th }}$ ed. Milton Park, Abingdon, Oxon: Routledge, 2014.

HASAN, R. Towards a paradigmatic description of context: systems, metafunctions, and semantics. Functional Linguistics, [s. l.], v. 1, n. 9, p. 1-54, 2014.

HOFFMAN, D. S. The Web of Hate: Extremists Exploit the Internet. Washington: AntiDefamation League, 1996.

IEDEMA, R. Multimodality, resemiotization: extending the analysis of discourse as multisemiotic practice. Visual Communication, [s. l.], v. 2, n. 1, p. 29-57, 2003.

JOHNSON, R. B.; ONWUEGBUZIE, A. J.; TURNER, L. A. Toward a Definition of Mixed Methods Research. Journal of Mixed Methods Research, [s. l.], v. 1, n. 2, p. 112-133, abr. 2007.

KARLOVA, N. A.; FISHER, K. E. A social diffusion model of misinformation and disinformation for understanding human information behaviour. Information Research, [s. l.], v. 18, n. 1, p. paper 573, 15 mar. 2013. 
KEVINTDFW. TOP 10 MELHORES TIRADAS DE JAIR BOLSONARO. 2018. Disponível em: https://www.youtube.com/watch?v=h1Q1O8Vj7Yo\&t=13s. Acesso em: 1 fev. 2020.

KASSAMBARA, A. ggcorrplot (V 0.1.3). 2020. Disponível em:

https://github.com/kassambara/ggcorrplot. Acesso em: 21 out. 2020.

KRESS, G. Multimodality: A Social Semiotic Approach to Contemporary Communication. London: Routledge, 2010.

LÉVY, P. A máquina universo: criação, cognição e cultura informática. Lisboa: Instituto Piaget, 1995.

LÉVY, P. Pela ciberdemocracia. In: MORAES, D. (Ed.). Por uma outra comunicação: mídia, mundialização cultural e poder. Rio de Janeiro: Record, 2012. p. 367-384.

LIMA-LOPES, R. E. DE; GABARDO, M. Ni una menos: A luta pelos direitos das mulheres na Argentina e suas representações no Facebook. Revista Brasileira de Linguística Aplicada, Belo Horizonte, v. 19, n. 4, p. 801-824, 2019.

LIMA-LOPES, R. E. DE; PIMENTA, I. \#Mulheresnofutebol: transitividade e avaliatividade na identificação de padrões sexistas. Humanidades \& Inovação, [s. l.], v. 4, n. 6, p. 116-132, 2017.

LIMA-LOPES, R. E. DE. O Conservadorismo como ideologia: Contribuições da ciência das redes para a linguística sistêmico funcional. Letras, Santa Maria, v. 28, n. 56, p. 43-69, 2018.

LIMA-LOPES, R. E. DE. Reflexões sobre as possíveis contribuições da linguística do corpus para a gramática sistêmico funcional: transitividade e classificação de processos.

Caletroscópio, [s. l.], v. 5, n. 9, p. 9-25, 2017.

LIMA-LOPES, R. E.; CÂMARA, M. T. Arco-íris na cruz: a multimodalidade no midiativismo em vídeos no YouTube. Policromias - Revista de Estudos do Discurso, Imagem e Som, Rio de Janeiro, v. 4, n. 2, p. 78-102, 2019.

MARTIN, J. R. English text: system and structure. Philadelphia: Benjamins, 1992.

MARTIN, J. R.; WHITE, P. R. R. The language of evaluation: appraisal in English. New York: Palgrave Macmillan, 2005.

MATTHIESSEN, C. M. I. M. Register in the round: diversity in a unified theory of register analysis. In: GHADESSY, M. (Ed.). Register analysis: theory and practice. New York: Pinter Publishers, 1993. p. 221-292.

MAZUI, G.; CALGARO, F. De capitão a presidente: conheça a trajetória de Jair Bolsonaro. [Rio de Janeiro], 2018, Portal G1. Disponível em: https://g1.globo.com/politica/eleicoes/2018/noticia/2018/10/28/de-capitao-a-presidenteconheca-a-trajetoria-de-jair-bolsonaro.ghtml. Brasília, 2018. Acesso em: 10 jun. 2020.

NAMISE, Daniel Kei. Dos Mitos ao "Mito": um estudo sobre o conceito de mito político na 
era da comunicação digital a partir da campanha presidencial de Jair Bolsonaro. 2019. 82 p. Trabalho de Conclusão de Curso (Curso de Graduação em Jornalismo) - Universidade Federal do Paraná, Curitiba, 2019. Disponível em: https://acervodigital.ufpr.br/handle/1884/67452. Acesso em: 19 jul. 2021.

O'REGAN, B.; MOMPEAN, A. R.; DESMET, P. From Spell, Grammar and Style Checkers to Writing Aids for English and French as a Foreign Language: Challenges and Opportunities. Revue francaise de linguistique appliquee, [s. l.], v. XV, n. 2, p. 67-84, 13 dez. 2010.

SILVA, R. O. Um mapa da «direita» no YouTube do Brasil através dos métodos digitais. 2018. 157 p. Dissertação (Mestrado em Ciências da Comunicação - Cultura Contemporânea e Novas Tecnologias) - Universidade Nova de Lisboa, Lisboa, 2018.

SOOD, T. Tuber (v. 0.9.9). Disponível em: https://github.com/soodoku/tuber. Acesso em: 20 out. 2020.

SOUZA, A. A. DE. Gradação: Força e Foco. In: VIAN JR, O.; SOUZA, A. A. de; ALMEIDA, F. A. S. D. P. (Eds.). A linguagem da avaliação em Língua Portuguesa. São Carlos: Pedro \& João Editores, 2011. p. 191-203.

SOVIGNON, S. J.; ROITHMEIER, W. Computer-mediated Communication: Texts and Strategies. CALICO Journal, [s. l.] v. 21, n. 2, p. 265-290, 14 jan. 2013.

STUBBS, M. British Traditions in Text Analysis: Firth, Halliday and Sinclair. In: STUBBS, M. Text and corpus analysis. London: Blackwell, 1996. p. 23-50.

THOMPSON, G. Introducing functional grammar. London/New York: Arnold/Oxford University Press, 2004.

THOMPSON, G.; ALBA-JUEZ, L. (EDs) Evaluation in context. Amsterdam; Philadelphia: John Benjamins Publishing Company, 2014.

VIAN JR, O. O sistema de avaliatividade e os recursos para gradação em Língua Portuguesa: questões terminológicas e de instanciação. DELTA: Documentação de Estudos em Linguística Teórica e Aplicada, [s. l.], v. 25, n. 1, p. 99-129, 2009.

VIAN JR, O.; LIMA-LOPES, R. E. DE. A perspectiva teleológica de Martin para a análise dos gêneros textuais. In: MEURER, J.; MOTTA-ROTH, D.; BONINI, A. (Eds.). Gêneros: teorias, métodos, debates. Campinas: Parábola, 2005. p. 29-45.

VIAN JR., O. A gradação da linguagem da literatura marginal. In: VIAN JR, O.; SOUZA, A. A. DE; ALMEIDA, F. A. S. D. P. (Eds). A linguagem da avaliação em Língua Portuguesa. São Carlos: Pedro \& João Editores, 2011. p. 205-212.

VOSOUGHI, S.; ROY, D.; ARAL, S. The spread of true and false news online. Science, [s. l.],v. 359, n. 6380, p. 1146-1151, 2018.

WEI, T.; SIMKO, V. R package "corrplot": Visualization of a correlation matrix. [S. l.: s. n.]. Disponível em: https://github.com/taiyun/corrplot. Acesso em: 20 out. 2020. 
WICKHAM, H. et al. Welcome to the Tidyverse. Journal of Open Source Software, [s. l.], v. 4, n. 43, p. 1686, 21 nov. 2019.

YOUTUBE. YouTube Official Blog. Disponível em: https://blog.youtube/. Acesso em: 1 fev. 2020.

Artigo submetido em: 24 abr. 2021

Aceito para publicação em: 23 jun. 2021

DOI: http://dx.doi.org/10.22456/2238-8915.113346 